\title{
CARACTERIZACIÓN NANOMECÁNICA DE LA ESTRUCTURA CELULAR Y ANATÓMICA DE Eucalyptus nitens Y SU RELACIÓN CON LA FRECUENCIA DE GRIETAS Y RAJADURAS EN MADERA REDONDA
}

\section{CHARACTERIZATION NANOMECHANICS OF WOOD CELL STRUCTURE AND ANATOMY IN Eucalyptus nitens AND ITS RELATION TO THE CRACKING AND FRACTURES IN ROUND WOOD}

\author{
Paulina Valenzuela $C^{1 \star}$, Cecilia Bustos $A^{2}$, Jean Pierre Lasserre ${ }^{3}$, William Gacitúa $E^{2}$
}

\section{RESUMEN}

El objetivo de este trabajo fue localizar el mínimo volumen en árboles de E. nitens que caracterice el comportamiento mecánico y anatómico para un muestreo no destructivo. Los árboles fueron cortados desde Mulchén y Yungay en la región del Bío-Bío. Se establecieron relaciones entre las propiedades nanomecánicas y la anatomía de la madera con el agrietamiento y las fracturas mayores (rajaduras). Se obtuvieron los parámetros razón de ductilidad $(E / H)$ y módulo de resiliencia $\left(u_{r}\right)$, medidos en la capa S2 y lamela media. Los resultados mostraron que el volumen representativo de los árboles de E. nitens se encontraban a 3 metros de altura desde la base de los árboles y desde el anillo 5 al 7. Al mismo tiempo, se logró establecer que la lamela media es más frágil que la capa S2 de la pared celular. Se determinó una relación entre la caracterización de los vasos y el nivel de agrietamiento de la madera. Esto es, a mayor área y diámetro de los vasos y a menor número de éstos, el nivel de agrietamiento fue mayor. Las grietas se concentraron principalmente en los primeros metros de altura del árbol, existiendo una buena relación con el $E / H$ de la lamela media, que mostró un cambio significativo a los 3 metros de altura. Las grietas en los anillos de crecimiento se centraron notoriamente en madera de temprana y en los anillos del 5 al 7 (desde médula a corteza).

Palabras claves: Propiedades mecánicas, nanoindentaciones, anatomía de la madera, ultraestructura de la madera, fracturas.

\begin{abstract}
The aim of this study was to define the minimum volumen in trees of E. nitens that characterize the mechanical and anatomy behavior for non-destructive sampling. The trees were cut from sites located in Mulchen and Yungay in the region of Bío Bío. Relationships were found between nanomechanical properties, wood anatomy and wood cracks and fractures. The response variables were the ductility ratio $(E / H)$ and the modulus of resilience $\left(u_{r}\right)$, in the S2 layer and middle lamella. The results showed that the representative volume, from $E$. nitens families is at 3 meter high from base of the trees and between the $5^{\text {th }}$ to $7^{\text {th }}$ annual rings. Also, it was established that the middle lamella is more brittle than the S2 layer. Relationship between the vessels morphology and the level of the wood cracking was observed. A high area of vessels, a high vessel diameter and a small frequency of them, resulted in high cracking. The cracks were mainly concentrated in the first meters of tree, were we found a good relationship of this response variable with the $E / H$ ratio for the middle lamella; there

\footnotetext{
${ }^{1}$ M. Sc. Ingeniero Civil en Industrias Forestales, Centro de Biomateriales y Nanotecnología (CBN). Universidad del Bío-Bío, Concepción, Chile. ${ }^{2}$ Profesor Asociado. Centro de Biomateriales y Nanotecnología (CBN). Depto. Ingeniería en Maderas, Facultad de Ingeniería, Universidad del Bío-Bío. Concepción, Chile.

${ }^{3}$ CMPC Forestal Mininco S.A. Concepción. Chile.

Autor correspondencia: ^nvalenzu@ubiobio.cl

Recibido: 29.08.2011 Aceptado: 29.04.2012
} 
was a significant change of this ratio at 3 meters high. Cracks were evident on earlywood and from the $5^{\text {th }}$ to the $7^{\text {th }}$ annual rings.

Keywords: Nanomechanical properties, nanoindentations, wood anatomy, wood ultrastructure, cracks.

\section{INTRODUCCIÓN}

En la década del 60 se efectuaron la primeras plantaciones en Chile, de la especie de Eucalyptus nitens (Valencia 2011) A través de los años, varias investigaciones se han realizado con el fin de conocer los atributos y características de esta especie (INFOR 2002, Mariani et al. 2005, Leandro et al. 2008, INFOR 2010). Las potencialidades del E. nitens se basan principalmente en la resistencia al frío y heladas y en su tasa de incremento anual promedio de $30 \mathrm{~m}^{3} /$ ha-año con rotaciones que van de 8 a 14 años (INFOR 2002). Estas particularidades generaron un alto potencial no tan sólo en la producción de pulpa sino que también en aplicaciones para productos manufacturados como paneles, chapas, molduras, entre otros. Las plantaciones de E. nitens en Chile son aproximadamente 168 mil hectáreas, centrándose desde la VIII región del Bío Bío a la X región de Los Lagos, generando interés en el óptimo aprovechamiento para proporcionarle mayor valor agregado (INFOR 2010).

La velocidad de crecimiento del E. nitens provoca tensiones de crecimiento, que cuando la madera es cosechada y procesada se presentan grietas en sus superficies, causando pérdidas para la industria maderera. Las grietas se presentan generalmente en la sección transversal de los árboles, mayoritariamente en la madera de temprana del duramen, perpendicular a los anillos de crecimiento (Gacitúa et al. 2007, Putoczki et al. 2007, Shelbourne et al. 2002, Lausberg et al. 1995). Estas grietas se generarían en la lamela media, constituida por una sustancia cementante que conecta las células de la madera y posee cantidades de lignina que van de 75 a 85\% en maderas duras, originando zonas débiles propensas a la formación de microgrietas (Putoczki et al. 2007, Donaldson 1995, Bodig y Jayne 1982). Las microgrietas se forman por la separación en la estructura de la pared celular causada por la fractura, la que puede ocurrir entre la lamela media y la capa S2. Las capas de las paredes celulares poseen módulo de elasticidad diferentes, generándose desplazamientos o deformaciones en éstas, al recibir cargas externas tales como las tensiones de crecimiento y por ende, la separación entre ellas a nivel ultraestructural (Putoczki et al. 2007, Gacitúa et al. 2007, Donaldson 1995).

La caracterización de las capas de la estructura celular se realiza por medio de la técnica de nanoindentación, que es utilizada para determinar las propiedades mecánicas de un material a nanoescala. Diferentes especies de maderas duras han sido nanocaracterizadas principalmente en la capa S2 y lamela media correlacionándolas con otras variables para obtener mayor información del comportamiento de la estructura celular (Wu et al. 2009, Gindl et al. 2004, Wimmer y Lucas 1997). Otra ventaja de esta técnica es que las probetas que se utilizan son pequeños cubos de $5 \mathrm{~mm}$, evitando de esta forma voltear los árboles al realizar la toma de muestras tales como la extracción de tarugos de incremento.

Para nanocaracterizar el E. nitens a través de muestreos no destructivos es necesario obtener el elemento de volumen representativo (EVR). Para materiales en general, el EVR corresponde al volumen más pequeño que puede realmente ser considerado como un medio continuo y todas las peculiaridades del material, sin embargo para el material madera, identificar este elemento es complejo debido a la heterogeneidad que presenta (Smith et al. 2003).

El E. nitens, al ser heterogéneo y anisótropo, sus propiedades pueden verse afectadas según su procedencia, tipo de suelo, edad, tipos de individuos e inclusive en el mismo individuo según la posición en la altura y diametralmente en el fuste (Mariani et al. 2005). Un estudio preliminar de $E$. 
nitens, proveniente de la VIII región, Chile, mostró que la frecuencia de vasos desde la médula hacia la corteza disminuye y que el diámetro de los vasos aumenta de médula a corteza (Leandro et al. 2008). Estos autores mencionan además, que el número de vasos aumenta de la base a la altura del árbol. Resultados similares fueron encontrados por Mariani et al. (2005), para esta misma especie, donde destacaron que sobre el 50\% de la altura comercial del árbol hubo mayor presencia de vasos.

Por otro lado, Shelbourne et al. (2002) señalan que, en datos no publicados por Lausberg et al. (1995), las grietas producidas en E. nitens disminuyen con la altura y se localizan en los anillos centrales. Estos resultados concuerdan con los obtenidos por Leandro et al. (2008), quienes observaron una mayor concentración de grietas en la base del árbol.

La heterogeneidad que se observa en esta especie con respecto a su anatomía y a la presencia de grietas obliga a encontrar un volumen que sea representativo de la microestructura local de la especie, que contenga un balance entre la complejidad de ésta y la intención de lograr un modelo simple que pueda ser tratado como un punto típico representativo de la especie en su conjunto.

De esta manera, el objetivo de este estudio fue definir el volumen representativo mediante caracterización nanomecánica de la estructura celular y anatómica del Eucalyptus nitens a lo largo del fuste y en los anillos de crecimiento que permita relacionarlo con la frecuencia de grietas y rajaduras presentes en esta especie.

\section{MATERIALES Y METODOS}

Doce árboles de la especie de E. nitens de 12 años fueron utilizados en este estudio. Seis de ellos

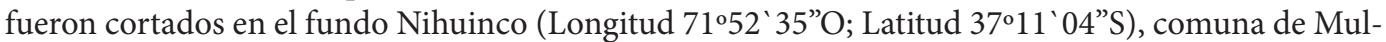

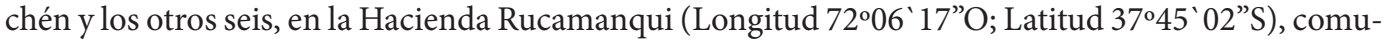
na de Yungay. Los espaciamientos de las plantaciones de ambos sitios fueron de $2 \times 3 \mathrm{~m}$. El suelo de fundo Nihuinco es del tipo arcilloso y los árboles se encontraban sin raleo, mientras que el fundo de Rucamanqui, posee un tipo de suelo de trumao y los árboles habían sido raleados. La altura media de los árboles en ambos sitios fue de aproximadamente $26 \mathrm{~m}$, con un DAP promedio de $20 \mathrm{~cm}$.

Se trabajó en dos etapas: la primera consistió en obtener el mínimo volumen con respecto a la altura del árbol y en una segunda etapa, se obtuvo el volumen representativo en los anillos de crecimiento, localizada en la altura representativa definida en la primera etapa.

\section{Obtención de muestras}

En la primera etapa, de los 12 árboles se cortaron rodelas de $5 \mathrm{~cm}$ de espesor a 11 alturas a lo largo del fuste equivalente a 1, 3, 5, 7, 9, 11, 13, 15, 17, 19 y 21 metros, desde la base hasta su altura comercial. De cada rodela se obtuvo un listón central de $1 \mathrm{~cm}$ de ancho y espesor, donde se identificó la dirección norte, que es donde se concentran principalmente las fuerzas externas tales como el viento. De cada listón se extrajeron 3 cubos de $3 \mathrm{~mm}$ de lado localizados en diferentes posiciones, cerca de la médula, en el centro y cerca de la corteza para la evaluación de las propiedades a nanoescala y para la caracterización anatómica.

En el análisis de grietas y rajaduras, se utilizó solo la mitad de las rodelas (semi-rodelas) que quedaron después de la obtención de los listones, presumiendo comportamiento simétrico de éstas en ambas mitades. Todas las rodelas fueron secadas al aire durante 30 días previo a su evaluación.

En la segunda etapa, se utilizó solo 1 semi-rodela correspondiente a la altura representativa obtenida en la etapa 1. Del lado norte de las semi-rodelas, 10 cubos de $3 \mathrm{~mm}$ de lado fueron obtenidos 
de cada anillo de crecimiento desde la médula a la corteza. La preparación de los cubos para caracterización anatómica y nanocaracterización se realizó de la misma manera que en la primera etapa. Dado que en esta especie las grietas se producen principalmente en madera de temprana (Leandro et al. 2008, Gacitúa et al. 2007), todos los cubos se extrajeron de esa zona.

\section{Nanoindentaciones}

Los cubos de $3 \mathrm{~mm}$ de lado fueron impregnados con resina epóxica para otorgarle mayor soporte mecánico a la estructura celular, obteniendo superficies planas y uniformes en los cortes microtómicos y para la posterior evaluación nanomecánica. Con un micrótomo de rotación se realizaron cortes de $250 \mathrm{~nm}$ de espesor en las superficies de los cubos, utilizando un cuchillo de vidrio y luego un cuchillo de diamante para otorgarle la calidad final adecuada para los análisis nanomecánicos. Las nanoindentaciones se realizaron mediante un nanoindentador Hysitron TI900, con una punta de diamante tipo cube corner, con una carga nominal máxima de $100 \mu \mathrm{N}$. Las respuestas nanomecánicas de la estructura celular de E. nitens, módulo elástico fue obtenido de la pendiente inicial de la zona de descarga en la curva carga-desplazamiento (Figura 1) y la dureza, fuerza aplicada con respecto al área proyectada, de la nanoindentación. El módulo de elasticidad reducido fue obtenido a través de la ecuación 1 .

$$
E_{r}=\frac{\sqrt{\Pi}}{2} \frac{S}{\sqrt{A}}
$$

Donde $E_{r}$ corresponde al módulo elástico reducido, resultante de la deformación elástica de la punta de diamante $(i)$ y de la muestra $(s), S$ es la pendiente de la curva de descarga $d P / d h$ en el inicio de la descarga y $A$ es el área de contacto entre el material y la carga máxima del indentador. Por otro lado, el módulo de elasticidad de la muestra $\left(E_{s}\right)$ se determinó a través de la expresión de Gindl et al. (2004):

$$
E_{r}=\left[\frac{1-v_{s}^{2}}{E_{s}}+\frac{1-v_{i}^{2}}{E_{i}}\right]^{-1}
$$

Donde $v_{s}$ y $v_{i}$ corresponden a la razón de poisson de la muestra (capa S2 o lamela media) y punta de diamante, respectivamente y $E_{i}$ al módulo de elasticidad de la punta de diamante.

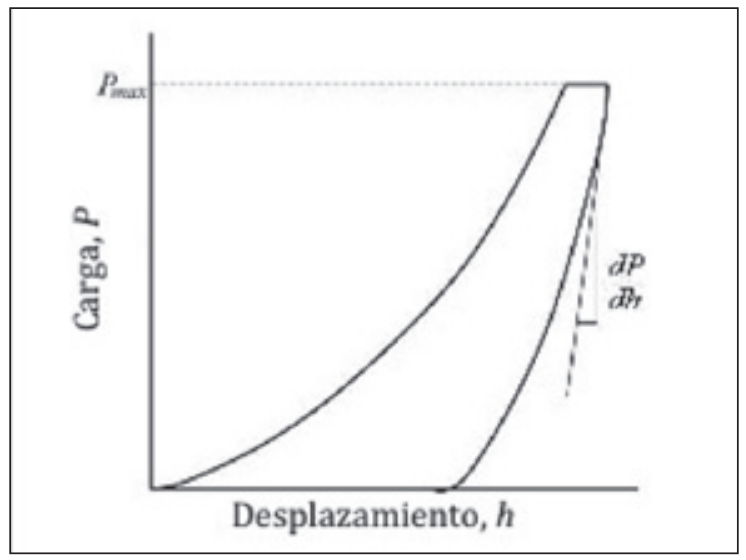

Figura 1. Curva carga-desplazamiento para ensayos de nanoindentación. 
La dureza fue obtenida dividiendo la carga máxima $P_{\text {máx }}$ por el área de contacto $A$. Otros parámetros utilizados para encontrar el volumen representativo fueron: la razón de ductilidad $E / H$ (Bolshakov y Pharr 1998), que indica si un material es más dúctil o es más frágil que otro (E/H mayor o menor respectivamente) y el módulo de resiliencia $\left(u_{r}\right)$, que se obtuvo del área bajo la curva esfuerzo-deformación hasta el límite proporcional mediante la siguiente expresión, que indica la energía absorbida por un material sometido a un esfuerzo en la zona elástica (Beer y Johnston 1992).

$$
u_{r}=\frac{\sigma_{y}^{2}}{2 E}
$$

\section{Análisis del porcentaje de grietas-rajaduras y morfología-número de vasos}

Las semi-rodelas fueron fotografiadas con cámara fotográfica digital para medir las grietas y rajaduras presentes en las muestras (Figura 2a). Por otro lado, se realizó un estudio de la geometría de los vasos de esta especie a través de un microscópio óptico que posee una cámara digital (Q-imaging). Las imágenes fueron tomadas en la sección transversal de los mismos cubos de $3 \mathrm{~mm}$ utilizados para las nanoindentaciones, tanto en la altura como para los anillos de crecimiento y fueron analizadas con ayuda del software Q-capture (Figura 3a).

El tratamiento de imágenes y mediciones de las grietas-rajaduras y de los vasos se realizó con el software Image-Pro Plus. Se determinó el porcentaje del área de grietas-rajaduras respecto del área total de la semi-rodela (Figura $2 \mathrm{~b}$ y $2 \mathrm{c}$ ). El número, área y diámetro de los vasos fueron medidos en un área de interés (AOI: area of interest) de $1 \mathrm{~mm}^{2}$, área representativa de la superficie total del cubo de $3 \mathrm{~mm}$ (Figura $3 \mathrm{~b}$ ).

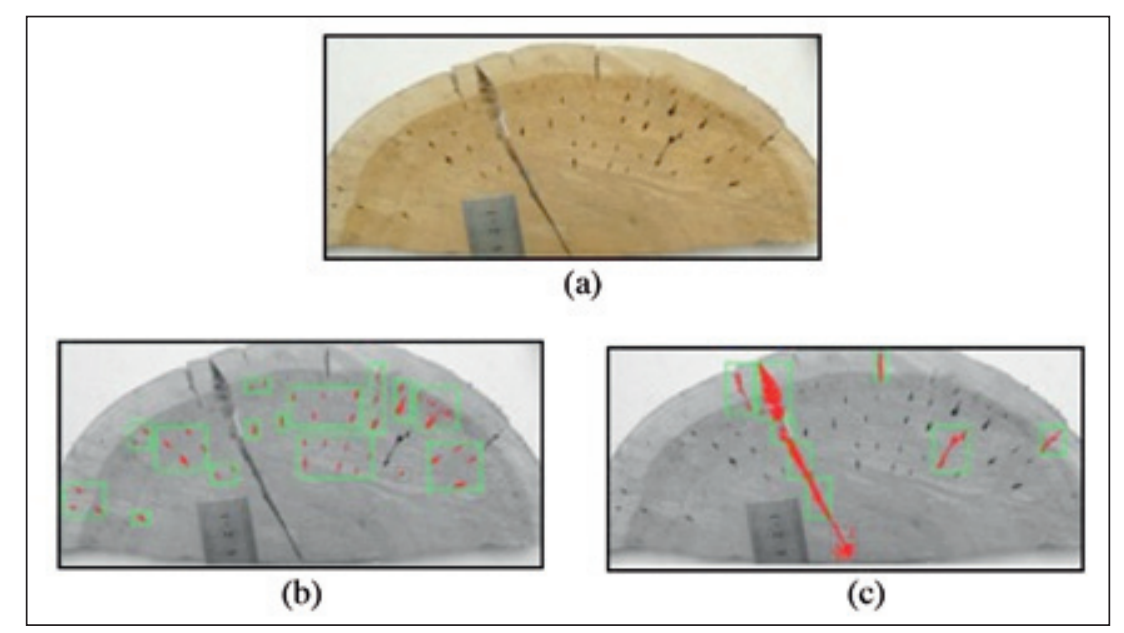

Figura 2. (a) Semi-rodela de E. nitens mostrando grietas-rajaduras en su superficie transversal (b) Medición de grietas-rajaduras con sotware Image-Pro Plus

(c) Medición de rajaduras con software Image-Pro Plus 


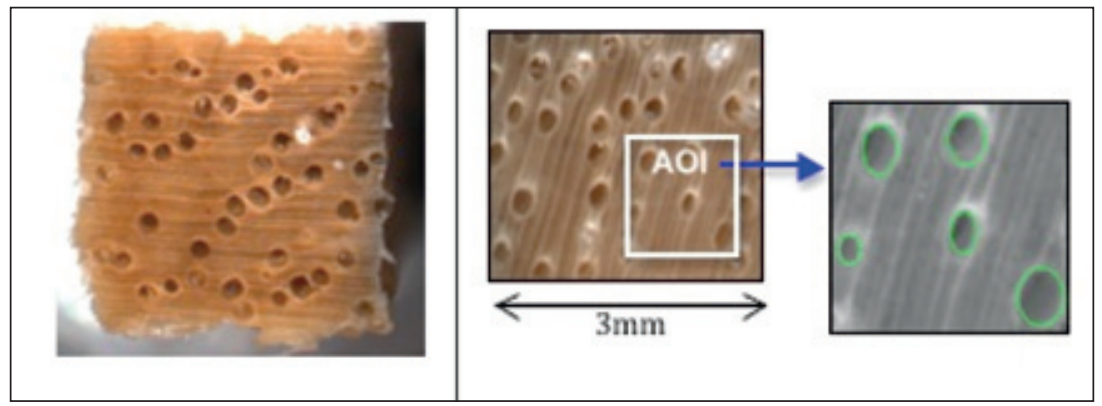

(a)

(b)

Figura 3. (a) Vasos presentes en una sección transversal de madera de temprana de E. nitens (b) Medición en área de interés $\left(\mathrm{AOI}: 1 \mathrm{~mm}^{2}\right.$ ) de vasos con software Image-Pro Plus.

\section{Análisis estadístico}

Los resultados fueron estudiados a través de un Análisis de Varianza (ANOVA) con un diseño factorial general. En la primera etapa los factores fueron: sitio (2 niveles) y altura (11 niveles). En la segunda etapa los factores fueron: sitio ( 2 niveles) y anillos de crecimiento (10 niveles). Los resultados se analizaron con un intervalo de confianza del 95\%. Se realizó un análisis de correlación en la etapa 2 de este estudio, específicamente en la anatomía con respecto a los anillos de crecimiento. Para indicar las relaciones existentes entre éstas, se determinó el coeficiente de determinación el cual mide el porcentaje de variación en la variable respuesta explicada por la variable independiente.

\section{RESULTADOS Y DISCUSIÓN}

\section{Etapa 1: Análisis con respecto a la altura por sitios}

\section{Módulo de elasticidad (E) y dureza (H)}

La figura 4 muestra los resultados promedios de $E$ y $H$ con respecto a la altura, de los árboles provenientes de Mulchén (Figura 4a y 4c) y de Yungay (Figura 4b y 4d) para la capa S2 y lamela media.

En general, el análisis de varianza (ANOVA) no mostró diferencias significativas en el $E$ de la capa S2 entre las alturas (valor- $\mathrm{p}=0.24$ ) y entre los sitios (valor- $\mathrm{p}=0.31$ ). Tampoco se evidenciaron diferencias significativas en la lamela media con respecto a las alturas (valor-p $=0.57$ ) y con respecto a los sitios (valor-p=0.53).

Con respecto a la dureza $(H)$ de la capa 2 , sólo se presentaron diferencias entre los sitios (valor$\mathrm{p}=0.048$ ) y no entre las alturas (valor- $\mathrm{p}=0.32$ ). Para la lamela media no hubo diferencias significativas entre los sitios (valor- $\mathrm{p}=0.8$ ) y entre alturas (valor- $\mathrm{p}=0.51$ ).

El sitio de Mulchén mostró un efecto significativo en la dureza con respecto a la altura (valor$\mathrm{p}<0.0027$ ) (Figura 4c). Mientras que ambos sitios, Mulchén y Yungay, afectaron significativamente el 
E (Figura 4a y 4b) y el $H$ (Figura 4c y 4d) de la pared celular (S2 y LM) con valor-p $<0.0001$. El sitio de Mulchén mostró un $E$ promedio de la capa S2 de 10.7 GPa mientras que el de Yungay fue de 10.1 GPa. Por otro lado, la lamela media promedió un $E=6.9 \mathrm{GPa}$ en el sitio de Mulchén y $E=7.3 \mathrm{GPa}$ en el sitio de Yungay. Para la $H$, la lamela media fue mayor respecto a la capa S2 de la pared celular con un valor promedio de $0.35 \mathrm{GPa}$ y $0.29 \mathrm{GPa}$ respectivamente en el sitio de Mulchén. En el sitio de Yungay la lamela media presentó un valor promedio de $H=0.34$ GPa y la capa S2 $H=0.28 \mathrm{GPa}$.

Estos resultados fueron similares a los reportados por Wimmer y Lucas (1997) quienes compararon las propiedades mecánicas de la capa S2 y lamela media para red Spruce (Picea rubens Sarg.) y a los reportado por Wu et al. (2009) quienes determinaron las propiedades mecánicas en la capa S2 para 10 diferentes madera duras, siendo la especie álamo (Populus spp.) la que más se asemeja a los valores obtenidos para el $E$. nitens. La diferencia en las propiedades nanomecánicas existentes entre la capa S2 y lamela media podría evidenciar la formación y aparición de microgrietas, cuando estén sometidas a fuerzas que podrían generar la separación entre ambas capas (Putoczki et al. 2007).

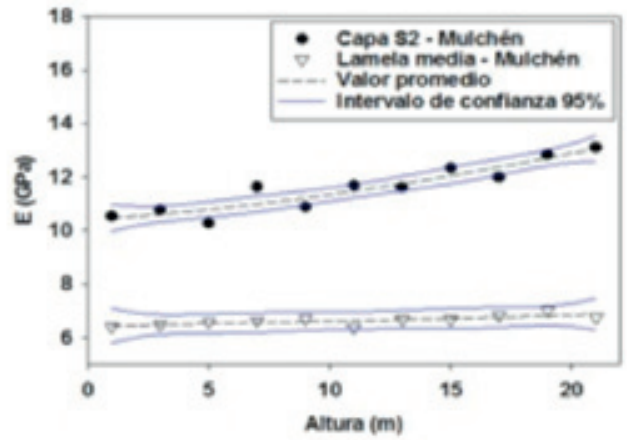

(a)

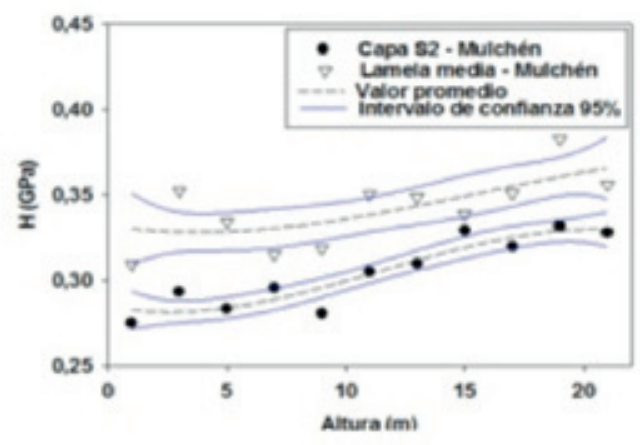

(c)

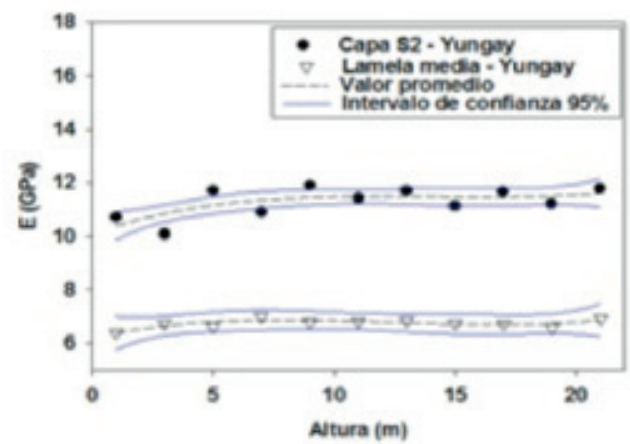

(b)

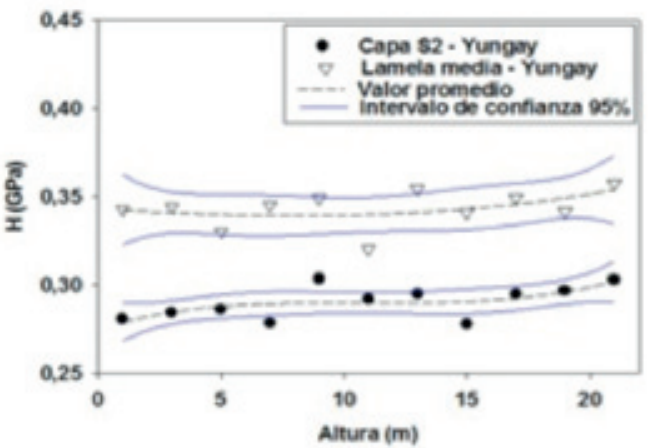

(d)

Figura 4. Promedio de las propiedades mecánicas de capa S2 y lamela media determinadas por nanoindentación de los seis árboles por sitio.

(a) E para sitio Mulchén (b) E para sitio Yungay

(c) H para sitio Mulchén (d) H para sitio Yungay.

La figura 5 muestra las marcas de nanoindentaciones dejadas después de un ensayo realizado en la capa S2 y lamela media de la especie de E. nitens utilizando una punta de diamante del tipo cube corner. 


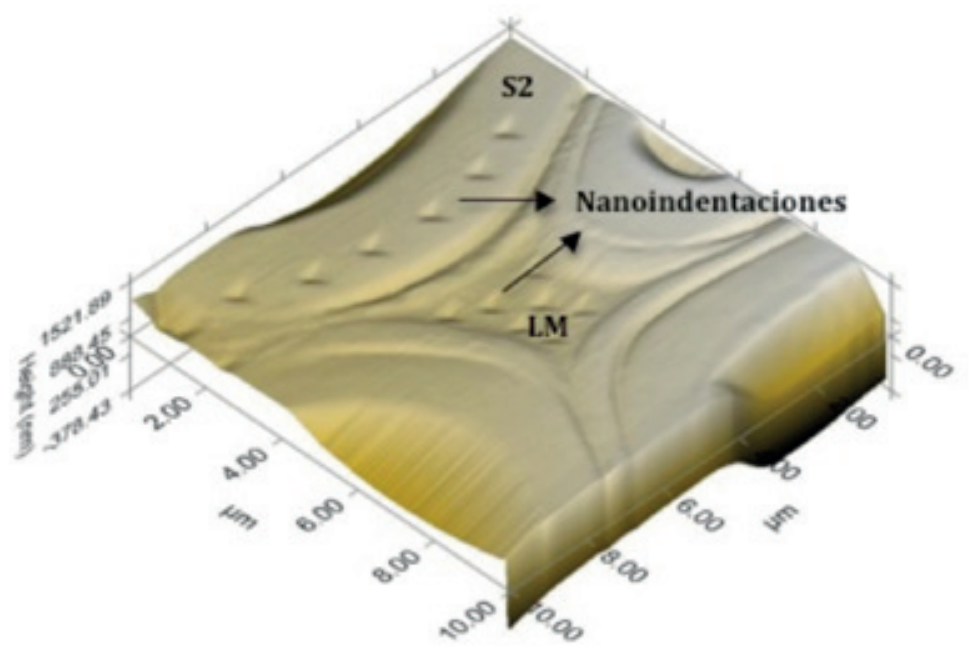

Figura 5. Vista 3D de nanoindentaciones en capa S2 y lamela media (LM).

\section{Razón de ductilidad (E/H) y módulo de resiliencia $\left(u_{r}\right)$}

La figura 6 muestra la razón de ductilidad $(E / H)$ y el módulo de resiliencia $\left(u_{r}\right)$ correspondiente al promedio de los seis árboles para el sitio de Mulchén (Figura $6 \mathrm{a}, 6 \mathrm{c}$ ) y los seis árboles del sitio de Yungay (Figura $6 \mathrm{~b}, 6 \mathrm{~d}$ ) con respecto a la altura. El análisis de varianza mostró que para la razón $E / H$ no existió diferencias significativas en la capa $S 2$ con respecto a los sitios (valor-p=0.34) y a las alturas (valor- $\mathrm{p}=0.49)$ y en la lamela media respecto a los sitios (valor- $\mathrm{p}=0.44$ ) y a las alturas (valor- $\mathrm{p}=0.51$ ).

Las razones de $E / H$ entre la capa S2 y la lamela media mostraron diferencias significativas (valor$\mathrm{p}<0.0001$ ) con valores aproximados de 40 y 20 respectivamente, tanto en el sitio de Mulchén como en Yungay. Esta razón $E / H$ ha sido utilizada para describir la rigidez de un material; materiales quebradizos tienen bajos valores de $E / H$, como el vidrio $(E / H=12)$ y materiales dúctiles tienen altos valores como el aluminio $(E / H=117)$ (Bolshakov y Pharr 1998). Dado los valores de $E / H$ obtenidos para la capa S2 (40) y lamela media (20), se desprende que la lamela media es frágil con respecto a la capa S2.

Por otro lado, para el $u_{r}$ el análisis de varianza no mostró diferencias significativas en la capa $\mathrm{S} 2$ con respecto a las alturas (valor-p=0.63). En la lamela media tampoco se mostraron diferencias significativas con respecto a los sitios (valor- $\mathrm{p}=0.66$ ) y con respecto a las alturas (valor- $\mathrm{p}=0.61$ ). Sin embargo, hubo diferencias significativas en la capa $\mathrm{S} 2$ respecto a los sitio (valor- $\mathrm{p}=0.03$ ).

Además, el $u_{r}$ presentó diferencias entre la capa S2 y la lamela media (valor-p<0.0001). En el sitio de Mulchén la lamela media tuvo un valor promedio de $u_{r}=0.001 \mathrm{GPa}$ y la capa S2 $\mathrm{u}_{r}=0.0004 \mathrm{GPa}$ y para el sitio de Yungay $u_{r}=0.0009 \mathrm{GPa}$ y $u_{r}=0.0004 \mathrm{GPa}$ respectivamente. Se observó que la lamela media fue mayor que la capa S2, indicando que la lamela media tiene la capacidad de absorber mayor cantidad de energía de deformación en comparación a la capa S2, lo que asociado a su bajo módulo de elasticidad y al alto valor de esfuerzo de fluencia medido con nanoindentaciones $\left(\sigma_{y s}=H / 3\right)$ se establece que la lamela media es un material resiliente (González-viña y Mancini 2003, Gibson y Ashby 1997). Dado que la madera es un compuesto natural, la lamela media se comporta como una matriz integrada en un constituyente tal como la capa S2 y por lo tanto, su capacidad de almacenar mayor cantidad de energía en comparación a la capa S2 permite que las fibras se puedan mover sin que sufran algún tipo de fractura hasta un cierto nivel de deformación. 


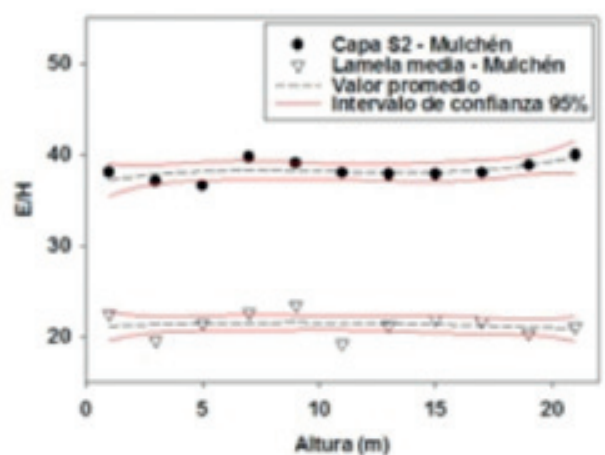

(a)

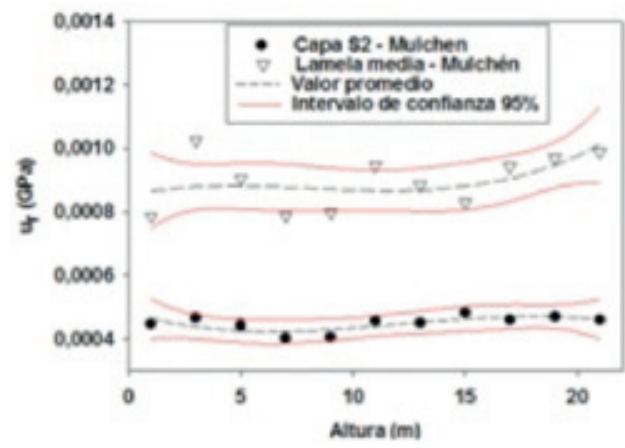

(c)

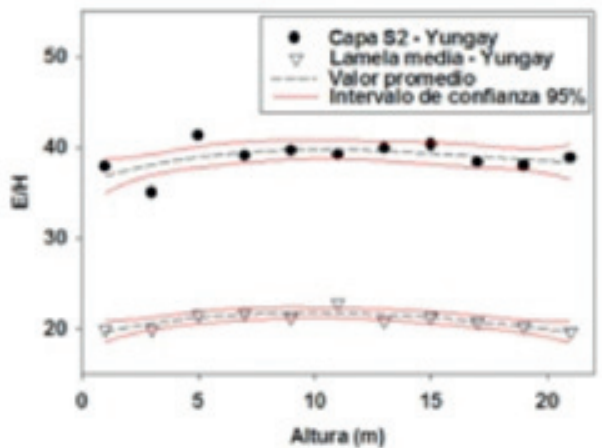

(b)

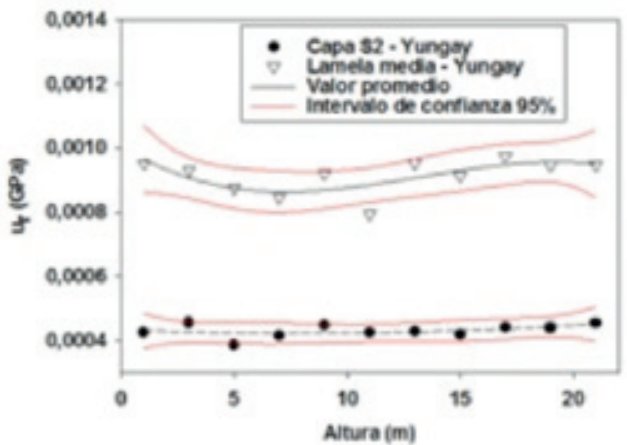

(d)

Figura 6. Promedio de la razón de ductilidad $\mathrm{E} / \mathrm{H}$ y $\mathrm{u}_{\mathrm{r}}$ de la capa $\mathrm{S} 2$ y lamela media para seis árboles por sitio.

(a) E/H para sitio Mulchén (b) E/H para sitio Yungay

(c) $u_{r}$ para sitio Mulchén $(d) u_{r}$ para sitio Yungay.

\section{Análisis de grietas y rajaduras}

$\mathrm{El}$ análisis del porcentaje de grietas y rajaduras se muestra en la figura 7a y Figura 7b, respectivamente. El análisis de varianza se aplicó al promedio de los seis árboles por sitio, que se muestran como puntos en los gráficos con respecto a la altura. El diseño factorial general mostró que el modelo es significativo para el porcentaje de grietas $(\mathrm{p}<0.0001)$ y el porcentaje de rajaduras $(\mathrm{p}<0.0001)$.

Existieron diferencias entre los sitios para el porcentaje de grietas $(\mathrm{p}=0.0022)$ y el porcentaje de rajaduras $(\mathrm{p}<0.0001)$. Los resultados señalaron que en el sitio de Yungay el porcentaje de grietas $(0.21 \%)$ y rajaduras $(0.73 \%)$ fueron mayores que los obtenidos en el sitio de Mulchén, $0.092 \%$ y $0.098 \%$ respectivamente.

Esto puede deberse a que en Yungay los árboles se encuentran con raleo logrando mayor espaciamiento entre los individuos. Esto favorece el aumento en el diámetro y en la altura de estos árboles, generando mayores tasas de crecimiento que producen tensiones internas que serían las causantes de las grietas. El diámetro de los árboles en el sitio de Mulchén fue de $19 \mathrm{~cm}$ y los del sitio de Yungay de $28 \mathrm{~cm}$. Por lo que, a mayor volumen del árbol mayor es el volumen de las grietas.

En cuanto al análisis del porcentaje de grietas y rajaduras con respecto a la altura, sólo se presentaron diferencias significativas en la variable respuesta grietas (valor- $\mathrm{p}<0.0001$ ) y no en la variable respuesta rajaduras (valor-p=0.14). El porcentaje de grietas disminuye desapareciendo hacia la 
zona apical, principalmente en el sitio de Mulchén. Este comportamiento también fue reportado por Shelbourne et al. (2002) y por Leandro et al. (2008) quienes observaron mayor concentración de grietas en la base del árbol. En el sitio de Mulchén también se observó que la $H$ en la lamela media, material más frágil, es menor en los primeros metros de los árboles que es donde se produce mayor agrietamiento. Mariani et al. (2005) mencionaron que la lignina se encuentra en mayor cantidad en el duramen que en la albura, disminuyendo hacia la zona apical. Por otro lado, Leandro et al. (2008) mostraron que existe mayor concentración de grietas en la base de los árboles producto de la duraminización. Esto podría explicar la mayor frecuencia de grietas en el sitio de Yungay donde los diámetros son prácticamente el doble respecto al sitio de Mulchén.
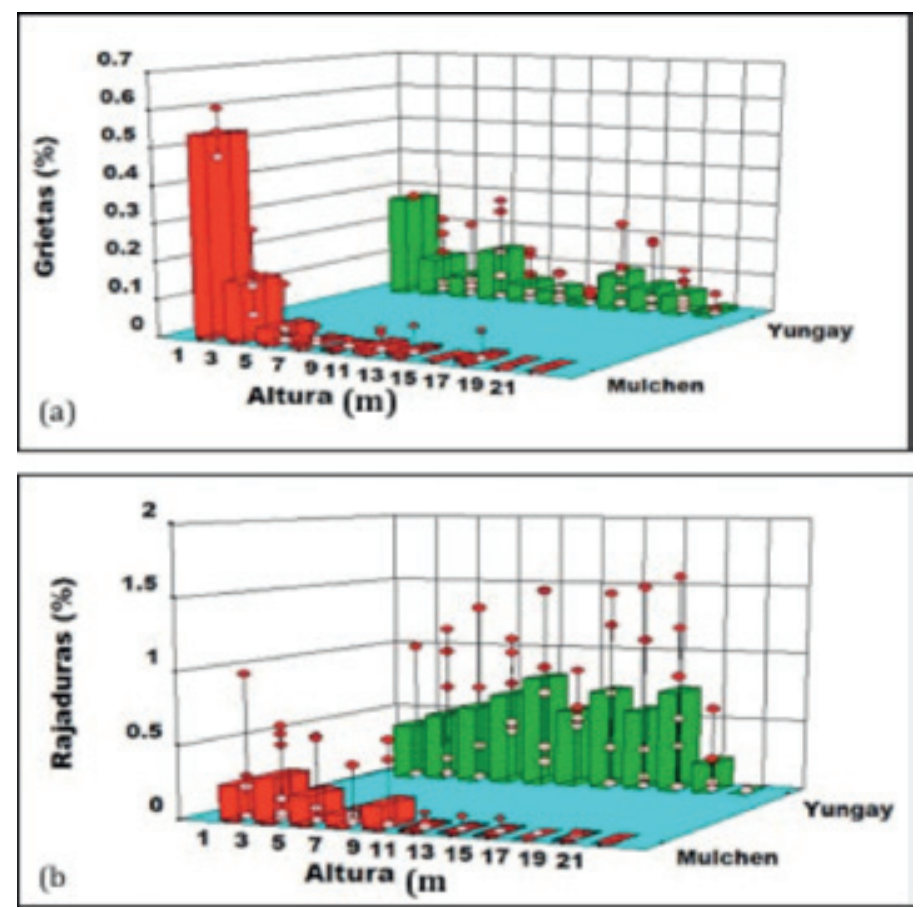

Figura 7. Superficie de respuesta interacción altura-sitio para el promedio de seis árboles por sitio (a) Porcentaje grietas en rodelas (b) Porcentaje rajaduras en rodelas.

\section{Análisis de la razón de ductilidad (E/H) en la lamela media para las primeras 5 alturas}

$\mathrm{El}$ análisis anterior de grietas mostró un mayor porcentaje en las primeras 5 alturas en el sitio Mulchén, y además, se estableció anteriormente en la razón $E / H$ que la lamela media es más quebradiza respecto de la capa S2 (Figura 6a y 6b), de este modo, se analizaron estos dos parámetros sólo hasta los 5 metros de altura, para la obtención del mínimo volumen del árbol.

La figura 8 muestra la razón de ductilidad $E / H$ de los primeros 5 metros de altura para el promedio de tres árboles. El ANOVA estableció que existen diferencias significativas en la razón de ductilidad entre los sitios $(\mathrm{p}=0.03)$ y en altura $(\mathrm{p}=0.04)$. En el sitio de Yungay, la lamela media se presentó más quebradiza que para el sitio de Mulchén y a la altura de 3 metros el valor de ésta para Yungay fue de $E / H=19.6$, lo que hace que la lamela media a esa altura sea mucho más frágil que a las otras alturas para ambos sitios. Esto indica que la altura más quebradiza donde se generan más grietas es a los 3 metros de altura. De este modo, existe una fuerte relación con la frecuencia de grietas donde el mayor porcentaje se concentró en las primeras alturas de los árboles. 


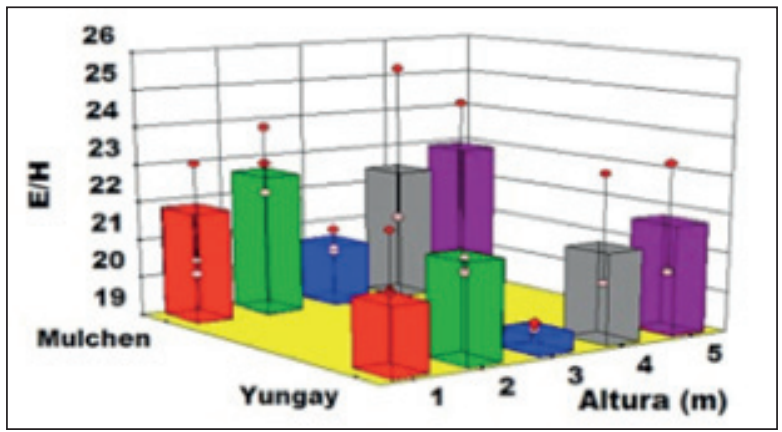

Figura 8. Superficie de respuesta del promedio de tres árboles para la razón E/H en la lamela media en los primeros 5 metros. Interacción altura-sitio.

Puntos indican valores individuales de los árboles.

\section{Análisis anatómico}

En la figura 9 se observan los resultados promedios de los seis árboles de E. nitens para el número, área y diámetro de vasos. La tabla 1 muestra los valores-p para el modelo, sitio y altura. El modelo fue significativo para el número, área y diámetro de los vasos. El mayor coeficiente de determinación del modelo se encontró con respecto al número de vasos $\left(\mathrm{R}^{2}=0.9083\right)$.

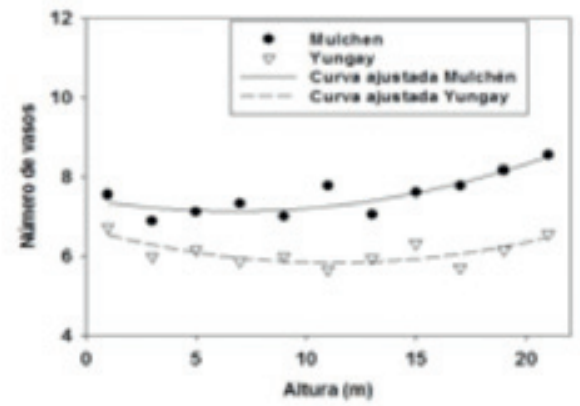

(a)

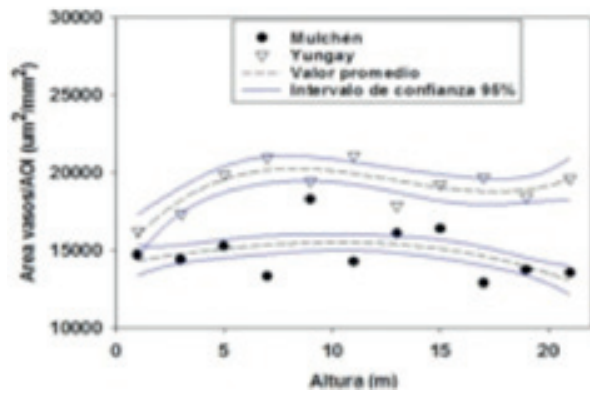

(b)

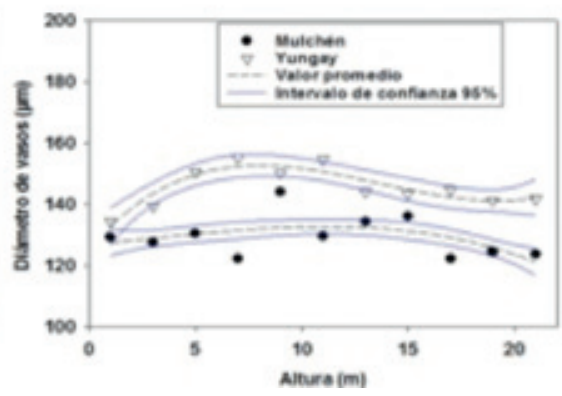

(c)

Figura 9. Promedio de los seis árboles en un área de interés (AOI: área of interés) (a) Frecuencia de vasos (b) Área de vasos por $\mathrm{mm}^{2}$ (c) Diámetro de vasos $(\mu \mathrm{m})$.

Se encontró un menor número de vasos en el sitio de Yungay con respecto al sitio de Mulchén (Figura 9a). Sin embargo, el área y diámetro de los vasos del sitio de Yungay, $18311 \mu \mathrm{m}^{2} / \mathrm{mm}^{2}$ y $143 \mu \mathrm{m}$ respectivamente fue mayor que el área y diámetro de los vasos de los árboles de Mulchén, 
$14544 \mu \mathrm{m}^{2} / \mathrm{mm}^{2}$ y $127 \mu \mathrm{m}$, (Figura 9b y $9 \mathrm{c}$ ). Esto es interesante, puesto que en este último sitio se presentaron los mayores porcentajes de grietas $0.21 \%$ en comparación a Mulchén $0.092 \%$. Esto podría explicar la mayor presencia de grietas en el sitio de Yungay. Dado que estos vasos producen una discontinuidad en el material sólido es que se forman concentraciones de esfuerzos en el borde de los vasos. Por lo tanto, la diferencia en el área de los vasos de Yungay respecto a Mulchén podría indicar mayor concentración de éstos debido a las tensiones de crecimiento desarrolladas en el árbol, las que se liberan originando microgrietas (grietas no visibles al ojo humano) y microfracturas induciendo agrietamientos que se propagarían a través de estructuras poliméricas más débiles, tal como la lignina presente en la lamela media (Gacitúa et al. 2007).

Tabla 1. Análisis de varianza de las variables respuestas con respecto a la altura para los vasos de E. nitens.

\begin{tabular}{lccc} 
& \multicolumn{3}{c}{ Valor- $^{*}(\alpha=\mathbf{0 . 0 5})$} \\
\cline { 2 - 4 } & Número vasos & Area vasos & Diámetro vasos \\
\hline Modelo & 0.0008 & 0.0044 & 0.0044 \\
Sitio & $<0.0001$ & $<0.0001$ & $<0.0001$ \\
Altura & 0.1944 & 0.4605 & 0.4291 \\
\hline$R^{2}$ & 0.9083 & 0.8675 & 0.8673 \\
$R^{2}$ ajustado & 0.8073 & 0.7218 & 0.7214 \\
\hline
\end{tabular}

* Valor-p $<0.005$ indica que los factores del modelo son significativos y Valor-p $>0.005$ indica que los factores del modelo no son significativos

Según el análisis de ductilidad a los 5 metros de altura y el análisis del porcentaje de grietas en estos árboles de E. nitens, la altura que mejor representó el comportamiento se localizó a 3 metros de altura. Por lo que los siguientes análisis fueron realizados en esa altura.

\section{Etapa 2: Análisis con respecto a los anillos de crecimiento en ambos sitios en la altura de 3 metros}

\section{Razón de ductilidad (E/H)}

La razón de ductilidad $E / H$ fue medido en un árbol debido a que las propiedades a nanoescala $E$ y $H$ medidas con respecto a la altura en la etapa anterior, no mostró variaciones significativas en los seis árboles, por lo que se presume que el comportamiento en los anillos de crecimiento es similar en los seis árboles.

La figura 10 muestra la razón de ductilidad $E / H$ en el sitio de Mulchén (Figura 10a) y el sitio de Yungay (Figura 10b) con respecto a los anillos de crecimiento desde médula (0) a corteza (12). El ANOVA no evidenció diferencias significativas en la razón de ductilidad con respecto al sitio y a los anillos de crecimiento.

Por otro lado, la lamela media mostró una razón de ductilidad de aproximadamente 20 y fue significativamente diferente a la razón de ductilidad de la capa S2 en un valor aproximado de 35, evidenciando que la lamela media es más quebradiza. 


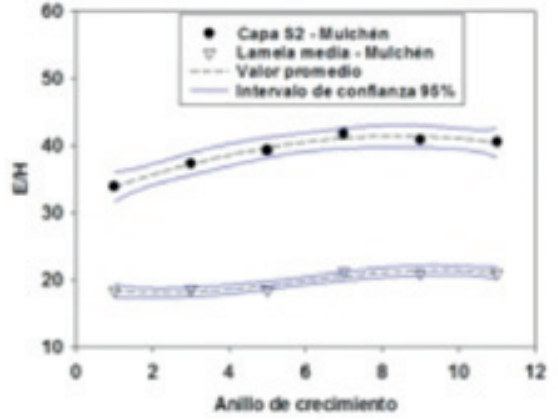

(a)

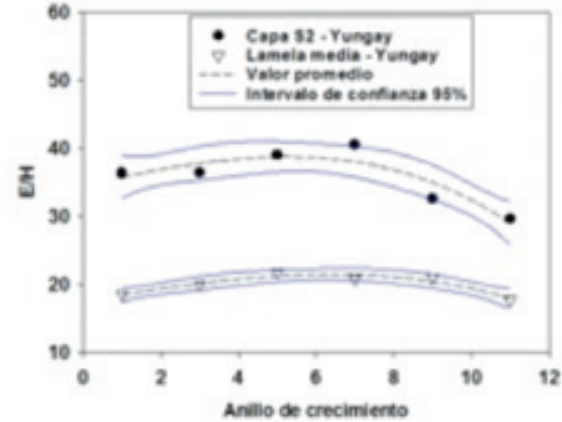

(b)

Figura 10. Razón de ductilidad E/H en capa S2 y lamela media (a) Sitio Mulchén (b) Sitio Yungay.

\section{Análisis de grietas}

Las grietas que se presentaron en los seis árboles, se producen mayoritariamente en madera de temprana (Figura 11), lo que concuerda con otras investigaciones (Leandro et al. 2008, Putoczki et al. 2007, Gacitúa et al. 2007). Además, se observó que las grietas se concentran en los anillos de crecimiento centrales, ocurriendo lo mismo en ambos sitios (Figura 11). Esto concuerda con lo publicado por Lausberg et al. (1995), quienes midieron el porcentaje de grietas en rodelas de $5 \mathrm{~cm}$ de espesor para E. nitens de 15 años de edad crecidos en Nueva Zelanda, reportando que este porcentaje se concentró en los últimos seis anillos del duramen (hacia la corteza), con un $89 \%$, es decir, se localizaron en la zona central de la rodela.

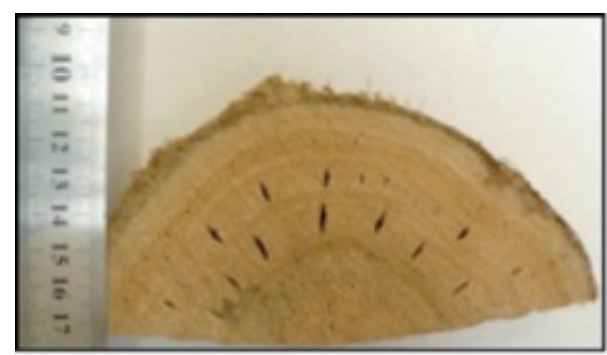

(a)

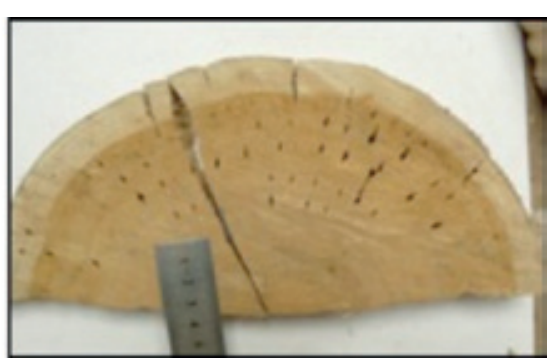

(b)

Figura 11. Imagen de grietas en anillos de crecimiento de E. nitens de 12 años en la altura de 3 metros (a) Sitio Mulchén (b) Sitio Yungay. Escala en cm.

La figura 12 muestra el promedio de las grietas visibles que se presentaron en madera de temprana en la mitad de las rodelas de los seis árboles. Los valores promedios de ambos sitios se intersectan aproximadamente en el anillo 5. En el sitio de Yungay el número de grietas se va incrementando y cae en el anillo de crecimiento 8 a diferencia del sitio de Mulchén, donde el número de grietas se vuelve constante en los anillos de crecimiento 4 a 8 y luego decrece. En general, el volumen de madera que mejor representa el factor promedio de agrietamiento, se localiza entre los anillos 5 y 7. 


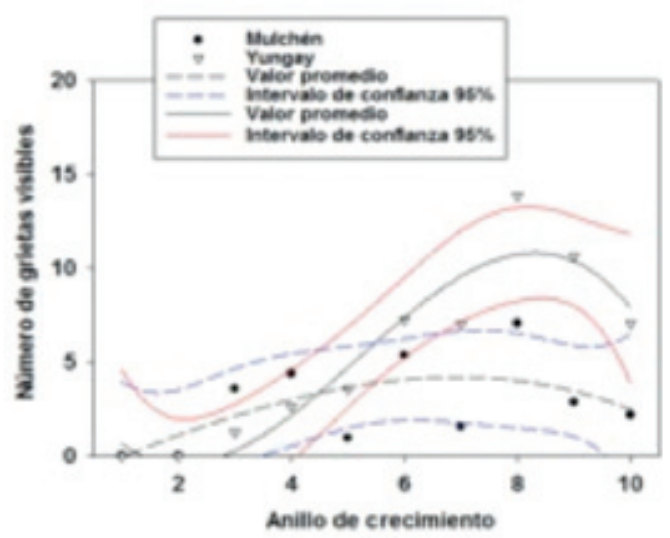

Figura 12. Promedio de los seis árboles de las réplicas 1 y 2 para el número de grietas visibles en el sitio de Mulchén y Yungay, desde médula (0) a corteza (10).

\section{Análisis anatómico}

El número y área-diámetro de los vasos se muestran en las figuras 13 y 14 respectivamente para el promedio de los seis árboles. La tabla 2 muestra los valores-p obtenidos para las variables respuestas con respecto a los anillos de crecimiento para los vasos del E. nitens. El modelo fue significativo para el número, área y diámetro de los vasos, donde el mayor coeficiente de determinación fue obtenido en el número de vasos $\mathrm{R}^{2}=0.9828$.

Tabla 2. Valores-p de las variables respuestas con respecto a los anillos de crecimiento para los vasos del E. nitens.

\begin{tabular}{|c|c|c|c|}
\hline & \multicolumn{3}{|c|}{ Valor $-p^{\star}(\alpha=0.05)$} \\
\hline & Número vasos & Area vasos & Diámetro vasos \\
\hline Modelo & $<0.0001$ & $<0.0001$ & $<0.0001$ \\
\hline Sitio & 0.0095 & 0.0293 & 0.0617 \\
\hline Anillo de crecimiento & $<0.0001$ & $<0.0001$ & $<0.0001$ \\
\hline$R^{2}$ & 0.9828 & 0.8483 & 0.9109 \\
\hline$R^{2}$ ajustado & 0.9636 & 0.8003 & 0.8827 \\
\hline
\end{tabular}

* Valor-p $<0.005$ indica que los factores del modelo son significativos y

Valor-p $>0.005$ indica que los factores del modelo no son significativos

El número de vasos disminuyó de médula a corteza en ambos sitios (Figura 13), esta tendencia fue diferente en lo que el área y diámetro de vasos se refiere, es decir, ambos aumentaron de médula a corteza (Figura 14). Estos resultados coinciden con los reportados por Leandro et al. (2008), Lausberg et al. (1995) y McKimm e Ilic (1987). 


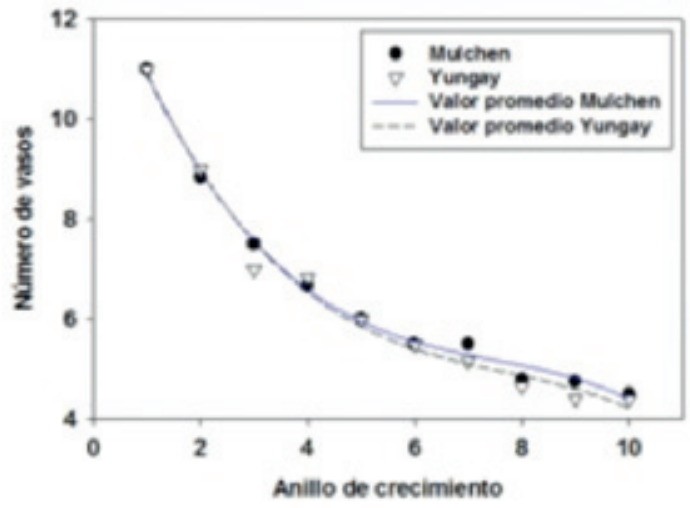

Figura 13. Número de vasos para el sitio de Mulchén y Yungay para el promedio de los seis árboles.

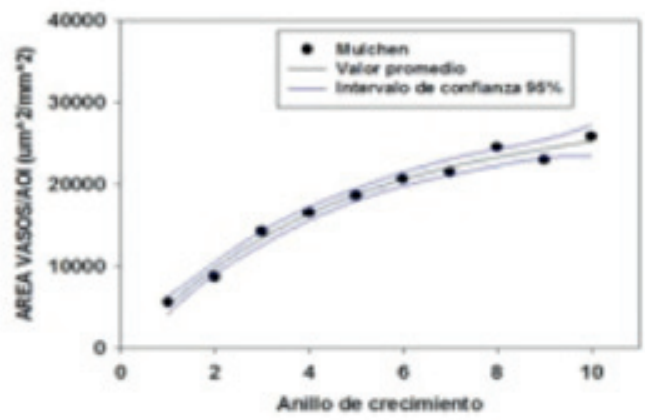

(a)

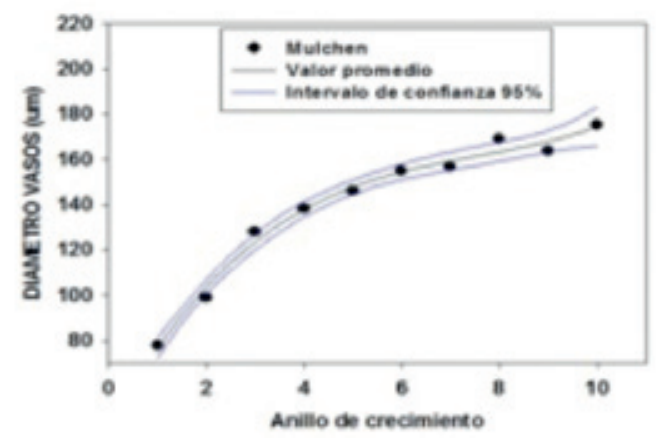

(c)

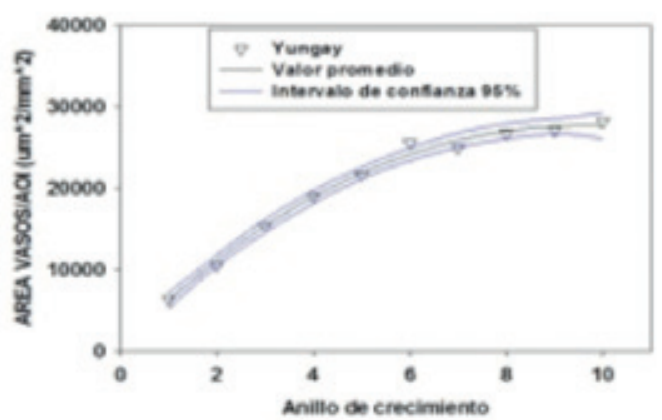

(b)

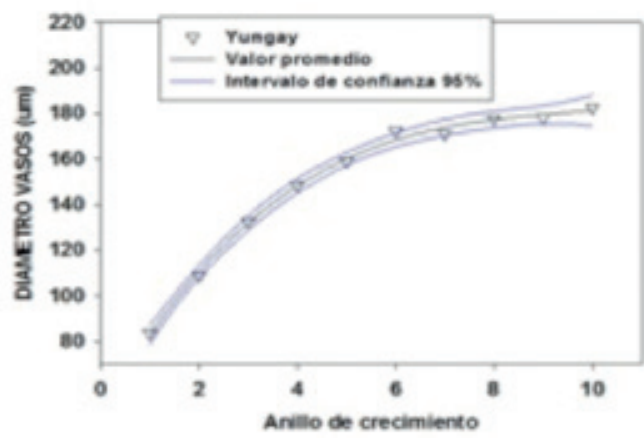

(d)

Figura 14. (a) Área de vasos por $\mathrm{mm}^{2}$ sitio Mulchén (b) Área de vasos por $\mathrm{mm}^{2}$ para sitio Yungay (c) Diámetro de vasos $(\mu \mathrm{m})$ para sitio Mulchén $(\mathrm{d})$ Diámetro de vasos $(\mu \mathrm{m})$ para sitio Yungay. Promedio de los seis árboles.

Todo material presenta un aumento de los esfuerzos cuando existen zonas discontinuas (agujeros) o imperfecciones en su estructura. De acuerdo a la fractura mecánica, los esfuerzos ocurren en la periferia de un agujero circular o elíptico, con un esfuerzo tangencial máximo mayor en agujeros con formas elípticas $\left(\sigma_{\max }=7 s\right)$ que en agujeros con formas circulares $\left(\sigma_{\max }=3 s\right.$; Sanford 2003). En el caso de la madera los vasos se presentan como zonas discontinuas con formas elípticas provocando 
concentración de esfuerzos elevadas alrededor de ellos que se originan a partir del centro de los vasos de la madera. El área y diámetro de los vasos en el sitio de Yungay se incrementan desde la médula a la corteza, hasta el anillo de crecimiento 7. Desde el anillo de crecimiento 8 hacia la corteza, el área y diámetro de los vasos tienden a ser constantes, esto puede indicar que las mayores concentraciones de esfuerzos alrededor de los vasos tienen su máximo en el anillo de crecimiento 7, lo que se relaciona con lo observado en la medición de grietas visibles donde se presenta una declinación en el anillo de crecimiento 8 decreciendo el número de grietas.

Según los resultados obtenidos y el análisis de varianza en las propiedades mecánicas a nanoescala, los vasos y porcentaje de grietas en estos árboles de E. nitens, los anillos de crecimiento que mejor representaron el comportamiento, fueron los números 5, 6 y 7.

\section{CONCLUSIONES}

La nanocaracterización, el análisis anatómico y la frecuencia de grietas y rajaduras presentes en de la especie de E. nitens de 12 años, permitió establecer que el volumen representativo se ubicó en la altura del metro 3 del árbol y en los anillos de crecimiento del 5 al 7.

Se definió que los parámetros que mejor se relacionan con el comportamiento de la estructura celular en relación con el agrietamiento, son la razón de ductilidad $E / H$ y el módulo de resiliencia $\left(u_{r}\right)$.

Los resultados de este estudio permiten concluir que la lamela media es más quebradiza que la capa S2 y que mayor área y diámetro de los vasos podrían provocar las grietas.

\section{AGRADECIMIENTO}

Los agradecimientos para el proyecto FONDEF D07i1101 de CONICYT por el apoyo financiero. Al Centro de Biomateriales y Nanotecnología (CBN) del Departamento de Ingeniería en Maderas de la Universidad del Bío-Bío por el equipamiento facilitado y a Forestal Mininco S.A. por facilitar la materia prima (E. nitens) para este estudio.

\section{BIBLIOGRAFÍA}

Beer, F.P.; Johnston, R. E. Jr. 1992. Mechanics of material. $2^{\text {a }}$ edición, McGraw-Hill Inc. New york.

Bodig, J.; Jane, B.A. 1982. Mechanics of wood and wood composites. Van Nostrand Reinhold Inc. USA.

Bolshakov, A.; Pharr, M. 1998. Influences of pileup on the measurement of mechanical properties by load and depth sensing indentation techniques. Journal of Materials Research 13(4):10491058.

Donaldson, L.A. 1995. Cell wall fracture properties in relation to lignin distribution and cell dimensions among three genetic groups of radiata pine. Wood Science and Technology 29: 51-63.

Gacitúa, W.; Ballerini, A.; Laserre, J.P.; Bahr, D. 2007. Nanoindentaciones y ultraestructura en madera de Eucalyptus nitens con micro y meso fracturas. Maderas. Ciencia y Tecnología 9(3): 259270 . 
Gibson, L.; Ashby, M. 1997. Cellular solids, structure and properties. $2^{\text {nd }}$ edition, Cambridge University press. United Kingdom.

Gindl, W.; Gupta, H.S.; Schöberl, T.; Lichtenegger, H.C.; Fratzl, P. 2004. Mechanical properties of spruce wood cell walls by nanoindentation. Applies Physics A 79: 2069-2073.

González-viña, W.; Mancini, H.L. 2003. Ciencia de los materiales. $1^{\text {a }}$ edición, Ariel S.A., Barcelona.

INFOR, Instituto Forestal. 2002. Disponibilidad de madera pulpable de Eucalyptus nitens en Chile 2001-2018. Informe Técnico No163. Ed. Instituto Forestal. Santiago Chile. 30 pp.

INFOR. Instituto Forestal. 2010. Anuario Forestal 2010. Instituto Forestal, Boletín estadístico No $128.134 \mathrm{pp}$.

Lausberg, M.J.F.; Gilchrist, K.F.; Skipwith, J.H. 1995. Wood properties of Eucalyptus nitens grown in New Zealand. New Zealand Journal of Forestry Science 25 (2): 147-163.

Leandro, L.; Ananías, R.; Cloutier, A.; Díaz-vaz, J.; Bermedo, M.; Sanhueza, R.; Laserre, J.P. 2008. Estudio preliminar de las grietas internas en anillos de madera inicial y su relación con características de la estructura anatómica y densidad en Eucalyptus nitens. Interciencia 33(11): 829-834.

Mariani, S.; Poblete, H.; Torres, M.; Fernández, A.; Morales, E. 2005. Caracterización física y química del Eucalyptus nitens con la altura. II Coloquio internacional sobre celulosa de eucalipto, 24-26 Mayo 2005. Concepción Chile. ATCP-Chile. 7pp.

McKimm, R.J.; Ilic, Y. 1987. Characteristics of the Wood of Young fast-grown trees of Eucalyptus nitens maiden with special reference to provenance variation. III: anatomical and physical characteristics. Austr Forest Res.17:18-28.

Putoczki, T.; Nair, H.; Butterfield, B.; Jackson S. 2007. Intra-ring checking in Pinus radiata D. Don: the occurrence of cell wall fracture, cell collapse, and lignin distribution. Trees 21:221-229.

Sanford, R.J. 2003. Principles of fracture mechanics. Pearson Education Inc. Prentice Hall. New Jersey. ISBN 0-13-092992-1. 404pp.

Smith, I.; Landis, E.; Gong, M. 2003. Fracture and Fatigue in Wood. John Wiley and Sons Southern Gate Chichester. England. ISBN 0-471-48708-2. 234pp.

Shelbourne, C.J.A.; Nicholas, I.D.; McKinley, R.B.; Low, C.B.; McConnochie, R.M.; Lausberg, M.J.F. 2002. Wood density and internal cheking of young Eucalyptus nitens in New Zealand as affected by site and height up the tree. New Zealand Journal of Forestry Science 32(3): 357-385.

Valencia, J.C. 2011. Introducción al nitens. Primer seminario regional INFOR "Eucalyptus nitens: Claves del mercado actual y potencial”. Valdivia. Corma. Los Ríos. Los Lagos. 28 Julio. 32pp.

Wimmer, R.; Lucas, B. 1997. Comparing mechanical properties of secondary wall and cell corner middle lamella in Spruce wood. IAWA Journal 18(1): 77-88.

Wu, Y.; Wang, S.; Zhou, D.; Xing, C.; Zhang, Y. 2009. Use of nanoindentation and silviscan to determine the mechanical properties of 10 hardwood species. Wood and Fiber Science 41(1): 64-73. 
